\title{
L-C MEASUREMENT ACQUISITION METHOD FOR AEROSPACE SYSTEMS
}

\author{
Stanley E. Woodard ${ }^{1}$ \\ NASA Langley Research Center \\ B. Douglas Taylor ${ }^{2}$ \\ Swales Corporation \\ Qamar A. Shams ${ }^{3}$ and Robert L. Fox ${ }^{4}$ \\ NASA Langley Research Center
}

This paper describes a measurement acquisition method for aerospace systems that eliminates the need for sensors to have physical connection to a power source (i.e., no lead wires) or to data acquisition equipment. Furthermore, the method does not require the sensors to be in proximity to any form of acquisition hardware. Multiple sensors can be interrogated using this method. The sensors consist of a capacitor, $\mathrm{C}(\mathrm{p})$, whose capacitance changes with changes to a physical property, p, electrically connected to an inductor, $\mathrm{L}$. The method uses an antenna to broadcast electromagnetic energy that electrically excites one or more inductive-capacitive sensors via Faraday induction. This method facilitates measurements that were not previously possible because there was no practical means of providing power and data acquisition electrical connections to a sensor. Unlike traditional sensors, which measure only a single physical property, the manner in which the sensing element is interrogated simultaneously allows measurement of at least two unrelated physical properties (e.g., displacement rate and fluid level) by using each constituent of the L-C element. The key to using the method for aerospace applications is to increase the distance between the L-C elements and interrogating antenna; develop all key components to be non-obtrusive and to develop sensing elements that can easily be implemented. Techniques that have resulted in increased distance between antenna and sensor will be presented. Fluid-level measurements and pressure measurements using the acquisition method are demonstrated in the paper.

\footnotetext{
${ }^{1}$ Senior Scientist, Structural Dynamics Branch, Structures and Materials Competency. Associate Fellow AIAA. s.e.woodard@larc.nasa.gov

${ }^{2}$ Design Engineer, Systems Engineering Competency

${ }^{3}$ Senior Electronics Engineer, Instrument Systems Development Branch, Aerodynamics, Aerothermodynamics and Acoustics Competency.q.a.shams@larc.nasa.gov

${ }^{4}$ Senior Electrical Technician, Technology Development and Integration Branch, Systems Engineering Competency

r.1.fox@larc.nasa.gov
} 


\section{Introduction}

Wireless methods are being developed for vehicle systems as a method for measurement acquisition ${ }^{1}$. To date, many of these systems are existing sensors physically connected to a power source, microprocessor and transmitters. Another method of acquiring measurements is the use of radio frequency identification (RFID) tags. RFID systems eliminate the need for physical connection to a power source. A RFID system consists of an interrogator (reader), a silicon chip electrically connected to an antenna coil and host computer. The silicon chip includes modulation circuitry and non-volatile memory. When the interrogator near the antenna coil transmits a time varying radio frequency wave, an alternating current (AC) voltage is created via Faraday induction 2,3 The AC voltage is rectified to create a direct current (DC) power source for the silicon chip. When the DC level reaches a critical threshold, then sufficient power exist to operate the chip. The chip can be electrically coupled to a sensor and measurements thus stored in the nonvolatile memory. When the RFID is activated, the contents of the nonvolatile memory can be transmitted to the reader. The distance for which RFID devices can be used is limited to approximately 15 in within the frequency range of $1-25 \mathrm{MHz}$ that is available for aerospace system 1 . Another measurement technique is the use of L-C sensors embodied as inductivecapacitive circuits.

A L-C sensor has a capacitor developed such that its capacitance, $\mathrm{C}(\mathrm{p})$, varies with changing physical properties, $\mathrm{p}$, which it is designed to measure. The capacitor is electrically coupled to an inductor, L, forming a $\mathrm{L}-\mathrm{C}(\mathrm{p})$ circuit $^{2,3}$. The electrical resonant frequencies of the $\mathrm{L}-\mathrm{C}(\mathrm{p})$ circuits change as their capacitance change. The capacitive changes are a result of changes in dielectric properties or geometric changes, which result from a physical property changing. An example would be that as a fluid fills the void between two capacitive plates, the dielectric properties between the plates and thus the capacitance changes. Piezoelectric materials (e.g., piezo-ceramics such as lead zirconate-titanate (PZT), or piezopolymers such as polyvinlydinofloruride (PVDF)) have electrical properties similar to capacitors. These materials develop electric polarization when force is applied along certain directions in them. The magnitude of polarization is proportional to the force (within certain limits). The capacitance varies as the polarization varies which suffices for measuring resulting strain from material deformation. Deformation can be due to either mechanical or thermal loading (pyroelectric effect). To date, the design of the
L-C circuits and the interrogation methods have limited applications to where the distance separating the L-C circuits and their interrogation systems are less than 15 $\mathrm{cm}$.

One example of a L-C sensor interrotation system is that presented by Konchin et al for interrogating a fluidlevel sensor ${ }^{4}$. The fluid-level sensor consists of two capacitive plates coupled to an inductor. The sensor uses the dielectric variation that results from a fluid filling the space between two capacitive plates. As the fluid level increases, the capacitance increases and thus the resonant frequency of the circuit decreases. The fluid-level sensor interrogator is a receiver that consists of an amplifier circuit with an inductor coil connected to the amplifier input and another inductor coil connected to the amplifier output. The coils are wound and are positioned with respect to each other to balance mutual inductance such that feedback between the coils is approximately zero. The balancing objective is to not create a positive input voltage to the amplifier. A voltage is applied to the amplifier thus powering the output inductor. When the fluid-level sensor is placed in proximity to the two coils, its inductor causes the amplifier output inductor to oscillate. The amplifier output inductor will cause the amplifier input inductor to oscillate thus creating a feedback system. The imput to the amplifier is then correlated to a value of fluidlevel. The limitation of this interrogation method is that the prefered separtion distance between the sensor and the interrogator is no more than $3.5 \mathrm{~cm}$.

Allen et al ${ }^{5,} 6$ discusses several methods of L-C sensor interrogation. In all cases, the sensors are within the perimeter of the antenna used for interrogation. In one method, an impedance analyzer applies a constant voltage signal to a transmission antenna. The frequency of the antenna is scanned across a predetermined range. When the antenna frequency is that of the L-C sensor frequency, the current across the antenna peaks. The frequency at which the current peaks is correlated to a physical property measurement. Another method presented by Allen et al is to use a transmission antenna and receiving antenna. White noise within a predetermined range is transmitted. Current is produced in the L-C sensor via Faraday induction. The current induced in the L-C sensor draws power from the transmission antenna. The receiving antenna receives the transmission antenna signal less the power absorbed by the L-C sensor which appears as a dip in the received response. Hence, the frequency of the response dip is correlated to the physical property measurement. Chirp interrogation is another technique discussed by Allen et al. A transmission antenna broadcast white noise and then is turned off after a 
predetermined amount of time. The white noise induces a current in the L-C circuit. The receiving antenna then listens to the $\mathrm{L}-\mathrm{C}$ response. A final method by Allen et al is to couple the transmission antenna to a tank circuit. The oscillator of the tank circuit varies the frequency of the transmission antenna. The frequency of the transmission signal is altered by the L-C sensor. A frequency discriminator is used to provide a signal from which the resonant frequency can be identified. 6

The key to practical use in vehicles is increased interrogation antenna-inductor separation distance and to facilitate multiple measurements having different dynamic characteristics. An L-C sensor serves as a means of acquiring power via Faraday induction, a sensor and a means of transmitting the measurement via the harmonic magnetic field created by the inductor. The distance at which the magnetic inductor response can be received is proportional to the strength of the magnetic field created in the inductor. The magnetic field strength is dependent upon the current in the sensor. Therefore, interrogation distance is also dependent upon the energy efficiently of the L-C sensor circuit. The higher the energy efficiency, the more current is created for the same level of power used by the interrogating antenna(e). The quality factor, $\mathrm{Q}$, is the gage for this efficiency. $Q$ is the ratio of reactance to DC resistance. A stronger magnetic field is created with higher Q.

The L-C measurement acquisition method presented in this paper can be used to acquire measurements even when the sensing element (inductor and capacitor) is embedded in material that is transmissive to the radio frequency energy that interrogates the sensing element. An advantage of this method is that the components for the method can be non-obtrusively added to the vehicle for which it is being used. An antenna can be produced as a metallic foil or as metal deposited on a thin dielectric film. Either aforementioned version of the antenna can be mounted to an existing bulkhead or other structural components. Furthermore, the antennae and L-C(p) elements can be fabricated using thin film deposition methods such as photolithography. The thin-film devices could be added to a vehicle during manufacturing. Other advantages of the method for vehicle applications are:

- Physical connection to a power source (i.e., lead wires) is not needed

- Physical connection to data acquisition equipment is not needed

- Multiple sensing elements can be interrogated using the single data acquisition channel (used for antenna).
- Key components can be developed as metallic foils or thin films (inductors, antennae, some capacitor types)

- No line-of-sight is required between antenna and L$\mathrm{C}(\mathrm{p})$ sensing element.

- The entire L-C(p) sensing element can be embedded in non-conductive material. For conducting material, the capacitive element can be embedded and the inductive element can be placed away from the surface of the conductive material.

- No specific orientation of sensing element with respect the antenna used to excite the sensing element is required except that they cannot be $90 \mathrm{deg}$ to each other.

- Easy to implement into existing vehicles/plants

- Easy to add new measurements. No wiring is required. All that is required is a partition of a RF bandwidth used in the measurement spectrum and frequency/measurement correlation table.

Following the introduction, the paper contains an overview of the measurement interrogation method. Examples of L-C sensors and respective test results will follow. Results will then be presented on methods being used to increase antenna $\mathrm{Q}$ and inductance $\mathrm{Q}$. Increasing inductor $\mathrm{Q}$ and antenna $\mathrm{Q}$ allows an $\mathrm{L}-\mathrm{C}(\mathrm{p})$ circuit to be interrogated at increased distance from the antenna. Techniques presented in this paper have resulted in measurements acquisition when antennae separation is $11 \mathrm{ft}$ using $1.5 \mathrm{w}$ of power applied to transmission antenna. Using a single antenna electrically switched from a transmitting to receiving antenna, an interrogation distance of $2 \mathrm{ft}$ has been achieved using $0.1 \mathrm{w}$ of power applied to the antenna.

\section{Overview of Measurement Interrogation Method}

Fig 1 shows a schematic of the L-C measurement acquisition method using a single radio frequency antenna and multiple L-C sensing elements. The components of the $\mathrm{L}-\mathrm{C}$ measurement system are a radio frequency antenna for transmitting and receiving $\mathrm{RF}$ energy; a processor for regulating the RF transmission and reception; and software for control of the antenna and for analyzing the RF signals received; and, L-C sensing elements. The sensing elements are capacitors, $\mathrm{C}(\mathrm{p})$, and inductors, $\mathrm{L}$, placed in parallel with their respective capacitors forming $\mathrm{L}-\mathrm{C}(\mathrm{p})$ circuits. The processor modulates the input signal to the antenna to produce either a broadband time-varying magnetic field or a single harmonic magetic field. The variable magnetic field creates an electrical current in the passive inductor-capacitor, $\mathrm{L}-\mathrm{C}(\mathrm{p})$, circuits as a result of Faraday induction. The circuits will electrically 
oscillate at resonant electrical frequencies that are dependent upon the capacitance and inductance of each circuit. The oscillation occurs as the energy is harmonically transferred between the inductor (as magnetic energy) and capacitor (as electrical energy). When the energy is in the inductors, the magnetic fields produced are single harmonic radio frequencies whose frequencies are the respective $\mathrm{L}-\mathrm{C}(\mathrm{p})$ circuits resonant frequencies. The antenna is also used to receive the harmonic magnetic responses produced by the inductors of the circuits. The receiving antenna can be the same antenna used to produce the initial broadcast of energy received by the $\mathrm{L}-\mathrm{C}$ circuit or another antenna can be used. When the same antenna is used, it must be switched from a transmitting antenna to a receiving antennna. A simple microprocessor can be used to identify the frequencies of the signals received by the antenna. The measured frequencies are then correlated to measurement of physical properties.

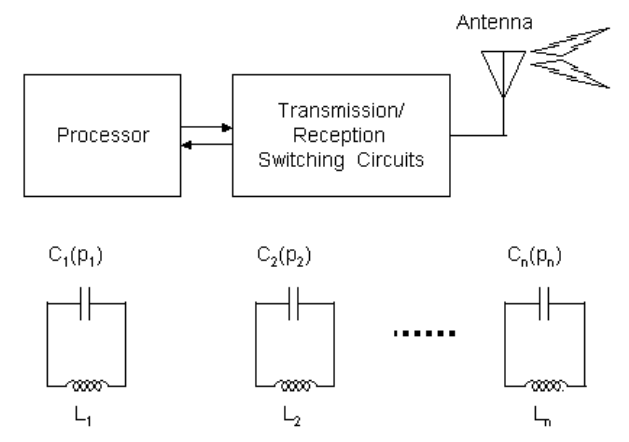

Fig 1 Schematic of L-C measurement acquisition method using a single radio frequency antenna and multiple L-C sensing elements

The L-C $(p)$ circuit responses are superimposed. The L$\mathrm{C}(\mathrm{p})$ circuits are designed (Fig. 2) such that their range of measurement frequencies do not overlap but the ranges are within a frequency range of the antenna. The range of resonant frequencies corresponds to physical property values that can be measured. An example would be that the lower frequency in the measurement band, $\omega_{i}(p)$, would correspond to the lower limit of a strain measurement. This method allows for any number of L-C sensing elements within the range of the antennae to be interrogated.

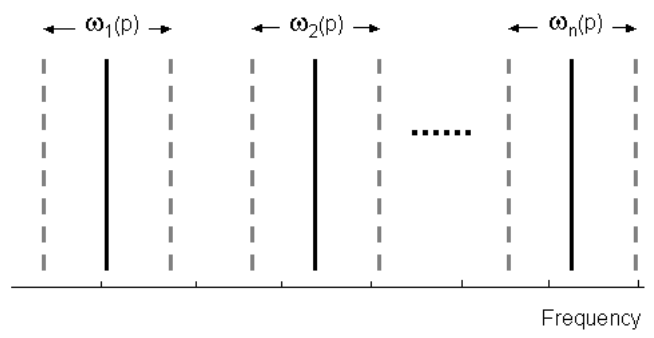

Fig 2 L-C sensor frequency measurement bands.

The interrogation method uses a scan-listen-compare technique. The interrogation logic is presented in Fig 3. The transmission and receiving antennae can be used or a single switching antenna can be used. Using two antennae provides a larger volumetric swath at which measurements can be taken which is approximately double that of a single antenna. The interrogation procedure goes as follows:

1. At the lower limit of a predetermined range, a radio frequency harmonic is transmitted for a predetermined time and then the transmission mode is swtiched off (i.e., the transmission antenna is turned off if two antennae are used or if a single antenna is used, it ceases transmission).

2. The receiving mode is then turned on (i.e., the receiving antenna is turned on if two antennae are used or if a single antenna is used, it begins receiving). The received response from the $\mathrm{L}-\mathrm{C}$ sensor is rectified to determine its amplitude. The amplitude, $A_{i}$, and frequency, $\omega_{1}$, are stored in memory.

3. The receiving mode is turned off and the transmission mode is turned on. The transmitted radio frequency harmonic is then shifted by a predetermined amount. The harmonic is transmitted for a predetermined time and then the transmission mode is turned off.

4. The receiving mode is then turned on. The received response from the $\mathrm{L}-\mathrm{C}$ sensor is rectified to determine it amplitude. The amplitude, $A_{i}$, and frequency, $\omega_{l}$, are stored in memory.

5. The current amplitude, $A_{i}$, is compared to the two previously attained amplitudes, $A_{i-1}$ and $A_{i-2}$. If the previous amplitude, $A_{i-1}$, is greater than the current amplitude, $A_{i}$, and also greater than amplitude prior to it, $A_{i-2}$, the previous amplitude, $A_{i-1}$, is the amplitude inflection. The amplitude inflection 
occurs when the excitation harmonic is equal to the resonant frequency of the L-C sensor. The amplitude, $\mathrm{A}_{\mathrm{i}-1}$, and the corresponding frequency, $\omega_{\text {l, }}$, are cataloged for the L-C sensor for the current frequency sweep. These values can be compared to the values aquired during the next sweep. If an amplitude inflection has not been identified, then steps 3 and 4 are repeated.

6. If amplitude inflection has been identified, the harmonic sweep continues to the next L-C sensor.

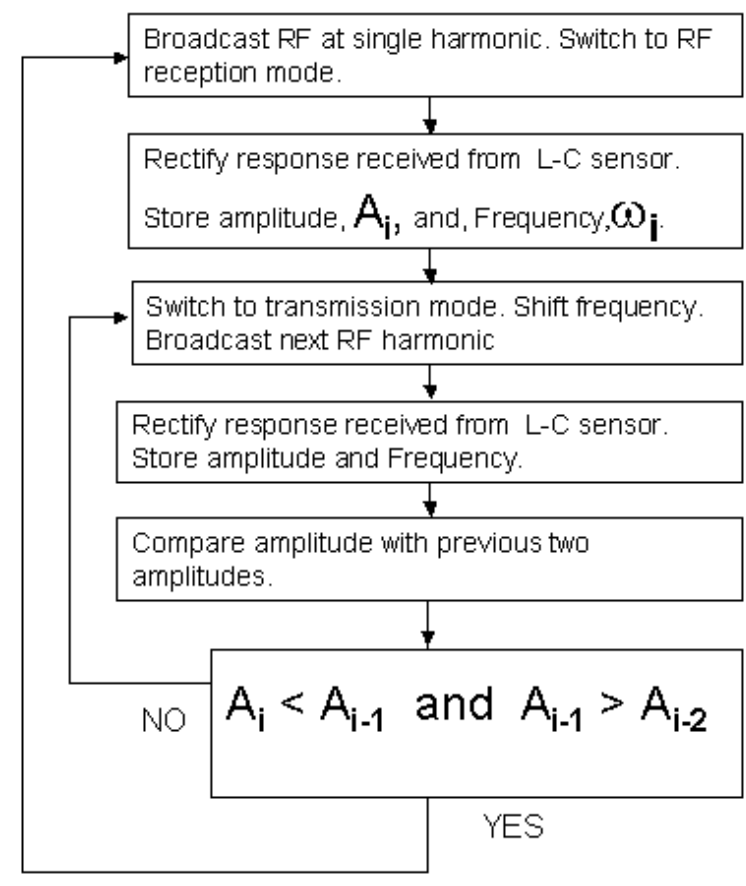

Interrogate next sensor

Fig 3 L-C measurement acquisition method interrogation logic

The initial frequency sweep can be used to identify and catalog all resonant peaks associated with all L-C sensors within its range of interrogation. The cataloged amplitudes and resonant frequencies for all L-C sensors can be used to reduce the sweep time for successive sweeps. For example, the next sweep to update each resonant frequency can start and end a predetermined proximity to the cataloged resonant and then skip to the next resonant range. The aforementioned capability allows L-C sensors to easily be added to measurement acquisition framework.
The sweep of a single frequency is used because it concentrates all energy used to excite the L-C sensor at that frequency. Fig 4 depicts the L-C sensor response amplitude as the excitation frequency approaches the sensor resonant frequency. During each frequency sweep for each sensor range, the current, $A_{i}$, and previous two amplitudes $\left(A_{i-1}\right.$ and $\left.A_{i-2}\right)$ and frequencies are stored. The amplitudes are compared to identify the amplitude inflection. The frequency at which the amplitude inflection occurs is the resonant frequency. The initial sweep is to ascertain all resonant frequencies and their corresponding amplitudes. Frequencies and amplitude values of successive sweeps can be compared to previous sweeps to ascertain if there is any change to measured property or if the antenna has moved with respect to the antenna. If the physical property has changed, the resonant frequency will be different from prior sweep. If a L-C sensor has moved with respect to the antenna, the amplitudes will be different (frequency will remain constant). The magnitude and sign of the difference can be used to determine how fast the sensor is moving and whether the sensor is moving toward the antenna or away from the antenna.

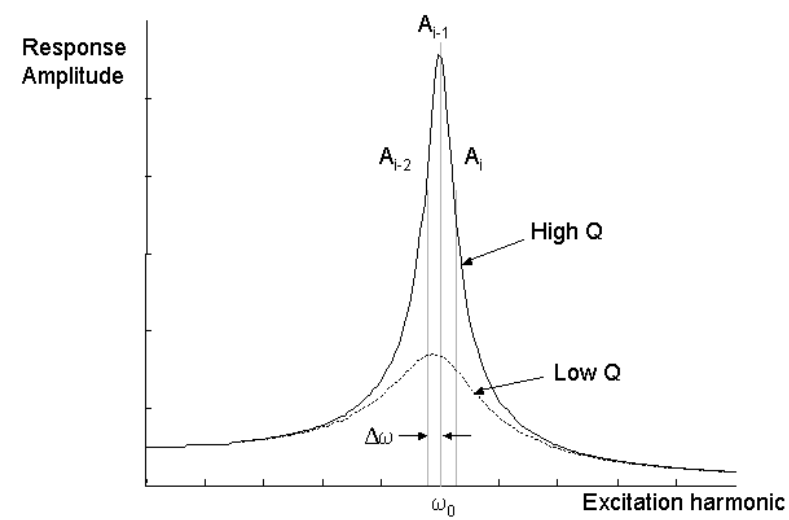

Fig 4. L-C sensor response to excitation radio frequency energy

\section{Examples and Application of L-C Sensors}

The L-C measurement acquisition method is applicable for a variety of measurements sensors. The method has been demonstrated for fluid-level and pressure measurements. Fig 5 shows an L-C(p) sensing element. The inductor (L) is formed as a spiral of copper. Interdigital electrodes have been used for the capacitor (C). The inductor and the capacitor have been deposited on a thin dielectric film. The sensing element has been used to measure pressure and fluid level of non-viscous fluids. A single antenna is used to power the L-C 
sensor and to receive its response. A wave generator is used to produce a single radio frequency harmonic. The excitation harmonic is tuned to the sensor's resonant frequency before force is applied. The harmonic is kept constant while eight pounds of force is applied to the L-C sensor shown in Fig 5. As the force is applied to the thin membrane, it causes the interdigital electrode separation distance to increase thus changing the resonant frequency. The response received by the antenna is shown in Fig 6 before and after the force was applied. The eight pounds of force resulted in the frequency response changing $0.5 \mathrm{MHz}$. When pressure is added, the response amplitude is decreased because the excitation frequency remained constant as the resonant frequency changed. The L-C sensor resonant frequency was measured before force was applied and with the force applied. However, having the excitation harmonic remain constant while the resonant frequency changed resulted in the lower response amplitude. This is also illustrated in Fig 4. The lower response level would reduce the distance at which changes in pressure level could be interrogated. The method presented in the prior section would eliminate the aforementioned shortcoming.

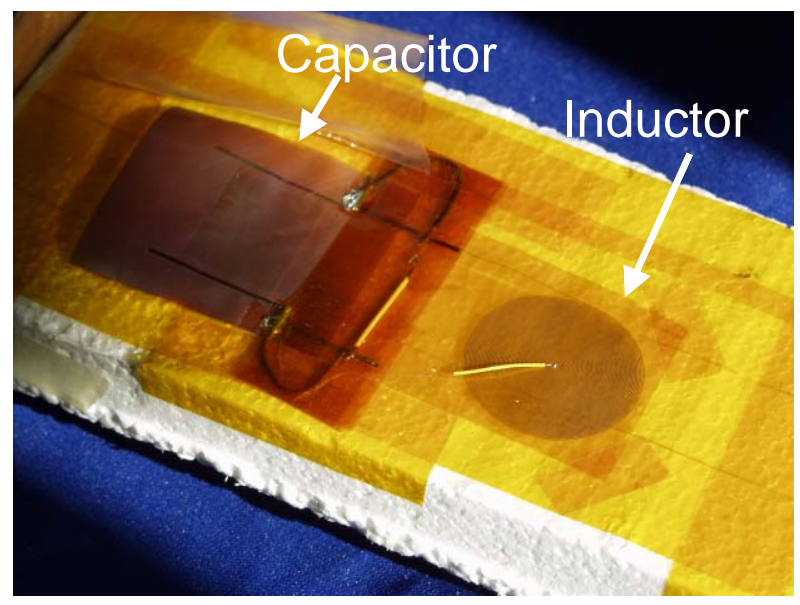

Fig 5 A L-C sensing element. The inductor (L) is formed as a spiral of copper. Inter-digital electrodes have been use for the capacitor $(C)$. The sensing element can be used to measure pressure and fluid level of non-viscous fluids.

A L-C fluid-level sensor is shown in Fig 7. The sensor consists of two capacitive plates electrically coupled to an inductor. As the fluid fills the void between the plates, the effective dielectric increases thus changing the resonant frequency. The fluid sensor was excited (powered) using the same method as the pressure sensor. Frequency measurements for a 9-inch fluidlevel sensor are shown in Fig 8. As the level increases, the frequency decreases. Fluid level was increased using 0.5 in increments. A fluid-level of 9 inches resulted in a frequency reduction of over $1 \mathrm{MHz}$ from that of the empty container. The fluid-level measurements were acquired manually using the procedure presented in the previous section.

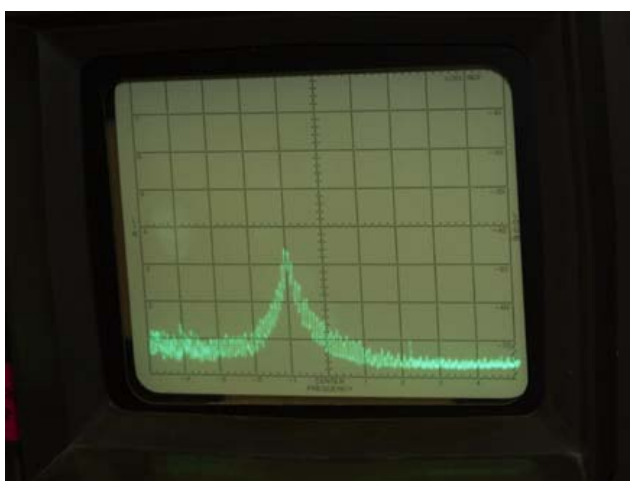

a. Frequency response when no pressure is applied to capacitor

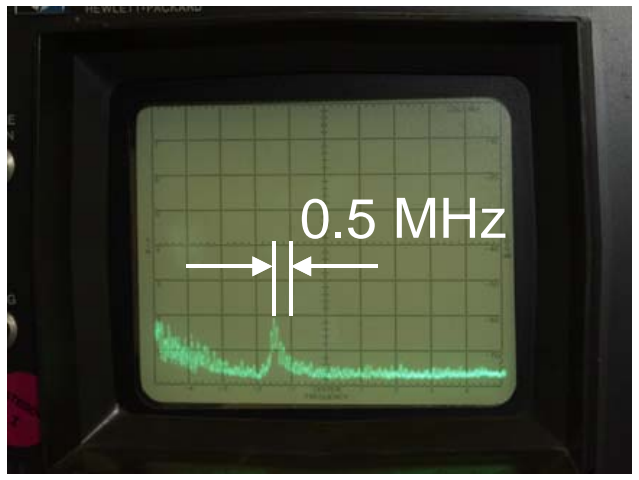

b. Frequency response when pressure is applied to capacitor

Fig 6 L-C sensing element (Fig 3) frequency response to applied pressure.

Fig 9 shows the RF response from multiple L-C(p) sensing elements. In this example, a broadband of radio frequency energy (1-10 MHz) excited the sensors. Each sensor has a predetermined frequency range, Fig 2 , which is correlated to its measurement range. The resonant peaks shown are for a fluid-level measurement, $\omega_{1}(p)$, position measurement, $\omega_{2}(p)$, and pressure measurement, $\omega_{3}(p)$. The range of frequencies (i.e., partition) for each measurement is annotated (arrows).

The examples demonstrate the L-C sensors and their respective measurements. To make this measurement method practical for vehicle applications, the distance between sensing elements and the interrogation antenna(e) needs to be more than $2 \mathrm{ft}$. The previous 


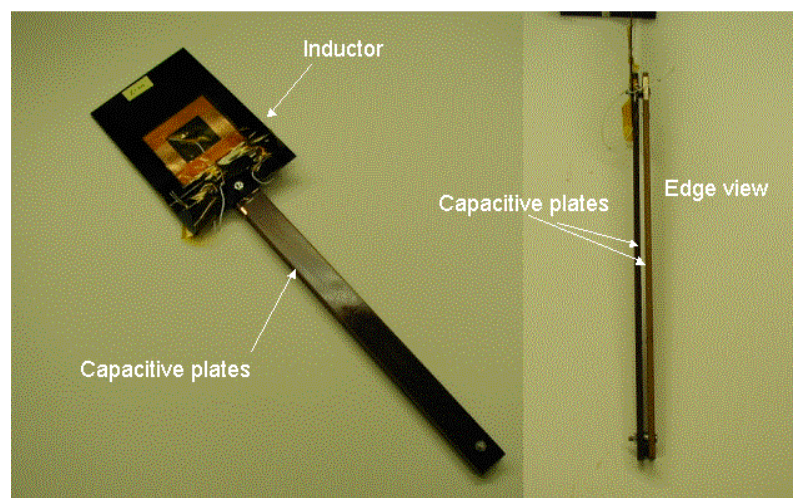

Fig 7 L-C Fluid level sensor

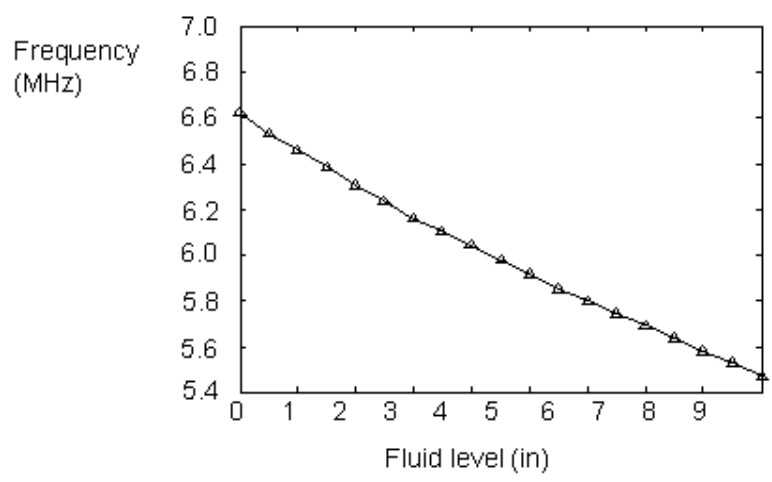

Fig 8 Electrical resonance variation with fluid level as measured by interrogation antenna

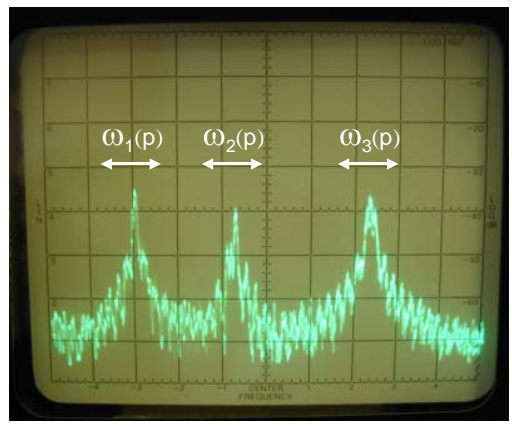

Fig 9 Frequency response of three measurements.

section discusses a method of concentrating energy into single harmonics to increase interrogation distance. Other methods to increase distance are to develop energy efficient antenna(e) and L-C circuits. These methods will be discussed in the next sections.

\section{Antenna Design Analysis}

Parametric measurements were performed to ascertain the influence of geometric properties on interrogation antenna effectiveness. The measurement objective is to develop design criteria to make antenna more energy efficient. To facilitate non-obtrusive use of the measurement system, the antenna are developed as either thin-film deposited on a dielectric membrane or thin foil which can be placed on any existing nonconductive surface. To ascertain the effect that geometry would have on the electrical properties, two features were considered: antenna width and antenna diameter. Fig 10a shows a thin copper foil antenna adhered to a Plexiglas plate. The antenna trace width is initially 2.0 in. In first study, the antenna outer diameter remained a constant 18.0 inches. The inner diameter is reduced and measurements were taken when the antenna trace was at the following widths: $2.0,1.5,1.0,0.5$, and 0.25 in. The inductance, DC resistance and $\mathrm{Q}$ were measured for each width. A current of $1 \mathrm{KHz}$ was used for the inductance measurements and the $\mathrm{Q}$ measurements.

Fig. 10b shows the other antennae used for parametric measurements. Six 0.5 in traces of copper foil are adherred to a Plexiglas plate. The outer diameters are $6,8,10,12,14$ and 16 inches. The coaxially cable was individually electrically connected to each trace. The inductance, DC resistance and Q were measured for each width. Resistance measurements are shown in Fig 11 for both parametric changes to antenna width and diameter. As seen in Fig 11a, DC resistance decreased with increased trace width. Resistance increased significantly as the width was reduced. The resistance doubled from $0.052 \Omega$ to $0.118 \Omega$ as the width was changed from 0.5 in to $0.25 \mathrm{in}$. The resistances of the wider traces were substantially less. For the traces wider than $1.0 \mathrm{in}$, the resistance decreased but not as much. Results from the DC resistance measurements (Fig 11b) of the diameter variations show that the resistance increased approximately linearly with diameter. The measurement results indicate that to develop low resistance antennae, a wide trace would result in less applied power loss due to lower resistance.

Inductance measurements are presented in Fig 12. The measurements have similar trends as the resistance measurements. Inductance increases are more pronounced for narrower traces. Inductance also increases approximately linearly with increasing diameter. Values of $\mathrm{Q}$ are presented in Fig 13. An antenna's electrical efficiency is dependent upon its $\mathrm{Q}$ (i.e., higher $\mathrm{Q}$ results in higher efficiency). The trace width has a significant effect on $\mathrm{Q}$. The increase of $\mathrm{Q}$ with increasing trace width is approximately linear. As the width is changed from 0.25 in to $2.0 \mathrm{in}$, Q changes by over a factor of 4 (Fig 13a). Changing outer diameter from 6 in to 16 in results in Q changing by less than 0.02 . 


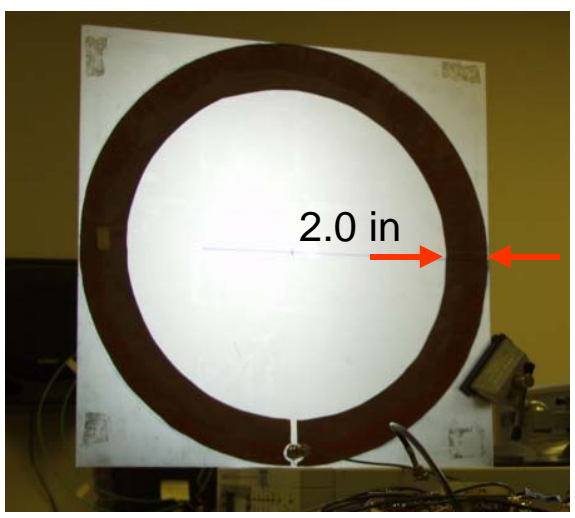

a. Antenna with constant outer diameter with variable width

Fig. 10 Antennae used for parametric studies

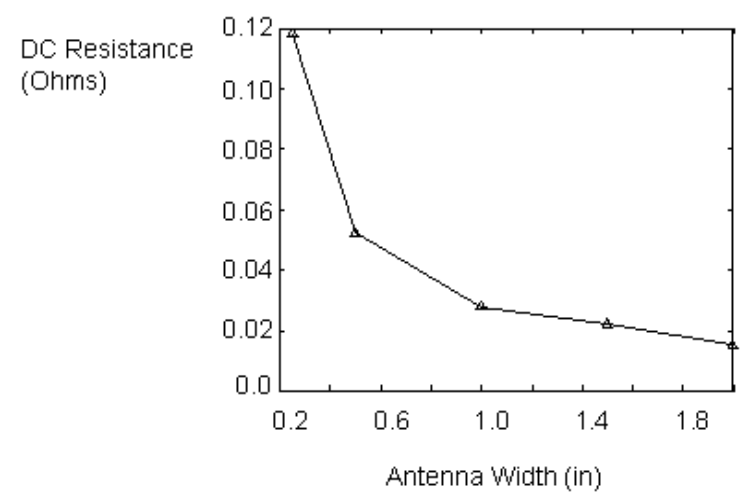

a. DC resistance variation with antenna width

Fig 11 DC resistance variation with antenna geometry.

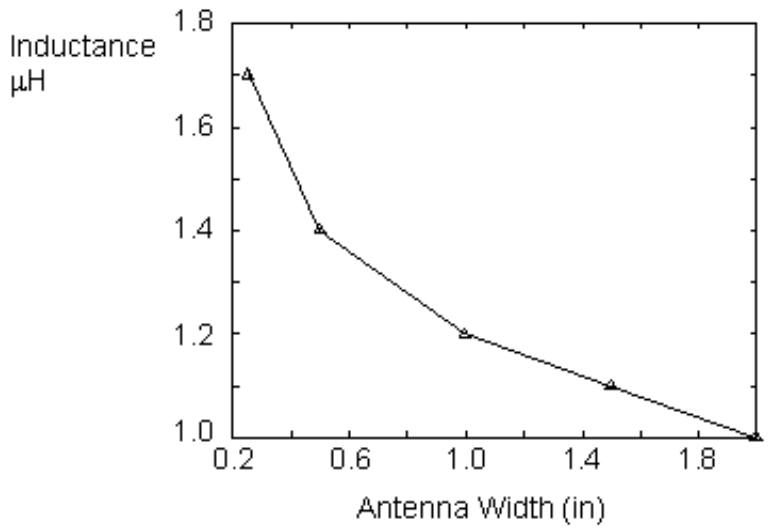

a. Inductance variation with antenna width

Fig 12 Inductance variation with antenna geometry

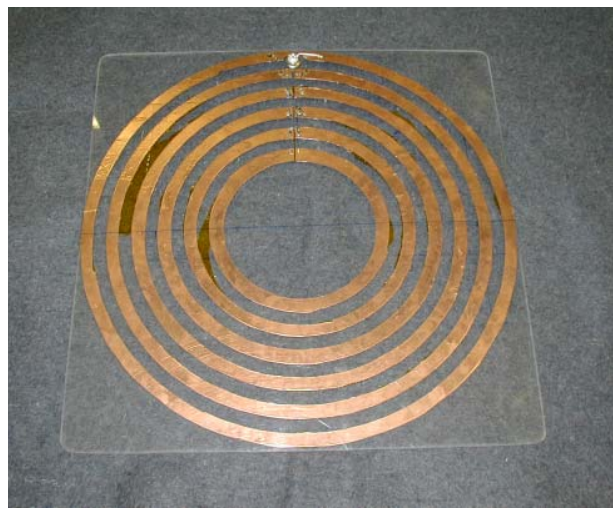

b. Antennae with 0.5 in wide trace of copper foil with various outer diameters

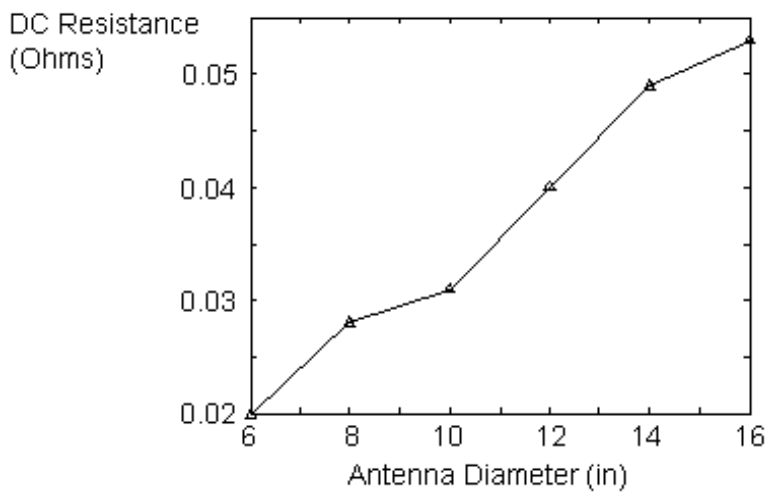

b. DC resistance variation with antenna diameter

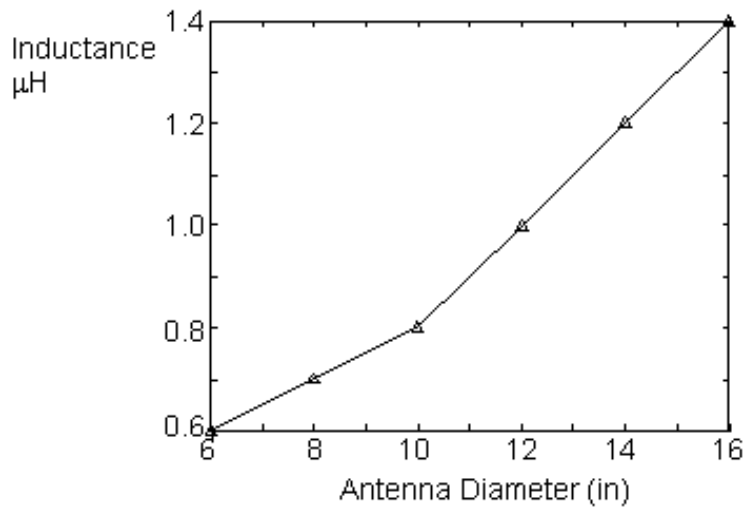

b. Inductance variation with antenna diameter 


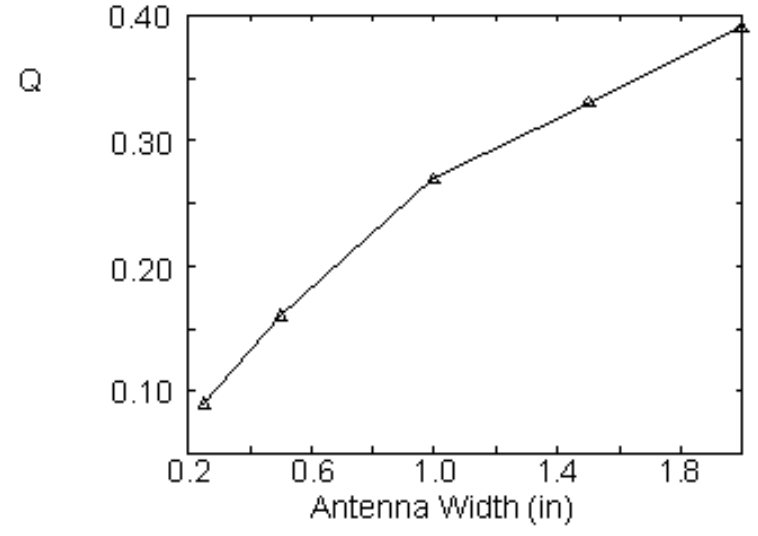

a. Q variation with antenna width

Fig $13 \mathrm{Q}$ variation with antenna geometry.

\section{Inductor Design and Analysis}

An antenna is basically an inductor. Hence, results for antenna parametric measurements can be applied for the development of inductors. Five square spiral inductors such as the 5 in inductor showed in Fig 14 were constructed to examine the effect that key design features such perimeter size and trace width had on inductance, DC resistance and Q. The inductor serves to relay the measurement. The distance at which the magnetic inductor response can be received is proportional the strength of the magnetic field created in the inductor. The magnetic field strength is dependent upon the current in the sensor. For the same applied energy, a lower resistance results in a higher current. Hence, to increase the range of the L-C sensors, the sensor elements should have as low resistance as possible.

Techniques used in the antenna analysis are used in the development of inductor. Specifically, using wider traces to reduce resistance. Fig 15 presents the effect of trace width on DC resistance. Three 3-inch square spiral inductors having widths of $0.02,0.25$ and 0.50 inches were used. The resistance of the 0.02 in trace is $9.4 \Omega$. Resistance for the 0.25 and 0.5 in traces are $0.055 \Omega$ and $0.023 \Omega$. The significantly lower resistance of the wider traces demonstrates the trace width is an effective design parameter. The effects of trace width and inductor perimeter size on $\mathrm{Q}$ are shown in Fig 16. The values $\mathrm{Q}$ for the 3 in square spirals are shown. The value of $\mathrm{Q}$ increases approximately linearly with increasing trace width. The 5 in square spirals $(0.25$ in and 0.05 in trace $)$ show similar results. The larger size square spiral

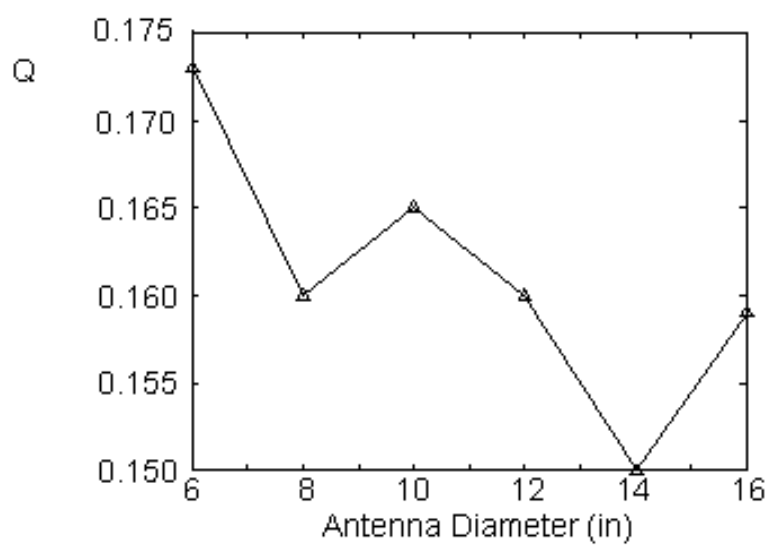

b. $\mathrm{Q}$ variation with antenna diameter

results in higher $\mathrm{Q}$ for same trace width. Comparison of the 5 in square with 0.25 in trace with the 3 in spiral with the 0.50 inch shows that increasing width can be used as a method of producing a higher inductor $\mathrm{Q}$.

To quantify effective range for measurement acquisition, the inductors were coupled to capacitors. Two measurement configurations were used. In the first configuration, a switching antenna (12 in loop) was used with a transmission power level of $0.1 \mathrm{w}$. An inductor with a 5 in $x 5$ in square spiral with 0.75 in trace coupled to a 504-pF capacitor achieved a $60 \mathrm{~dB}$ response at 25 in distance from the antenna. The inductor with the 3 in $\times 3$ in square spiral with 0.25 in trace coupled with a 826-pF capacitor achieved a $-60 \mathrm{~dB}$ response at 22 in distance from antenna. In second measurement configuration, a transmission antenna (18 outer in diameter and 0.5 in trace) and receiving antenna (wire loop 24 in using 12 gauge copper wire) are used. They are positioned $11 \mathrm{ft}$ apart. The antennae are operated such that when the transmission antenna is powered on to excite the L-C elements, the receiving antenna is off. The transmission antenna used $1.5 \mathrm{w}$ of power. When the transmission antenna is switch off, the receiving antenna is powered on allowing it to receive the L-C element response. In this configuration, the sensing elements presented in the section "Examples of L-S Sensors" could be interrogated anywhere in a volume approximated by a cylinder whose longitudinal axis runs between the antenna centers and with diameter approximately $4 \mathrm{ft}$. The length of the cylinder was the separation distance between the antennae. When the antennae were separated by $9 \mathrm{ft}$, the same sensing elements could be interrogated using $1.0 \mathrm{w}$ of power. 


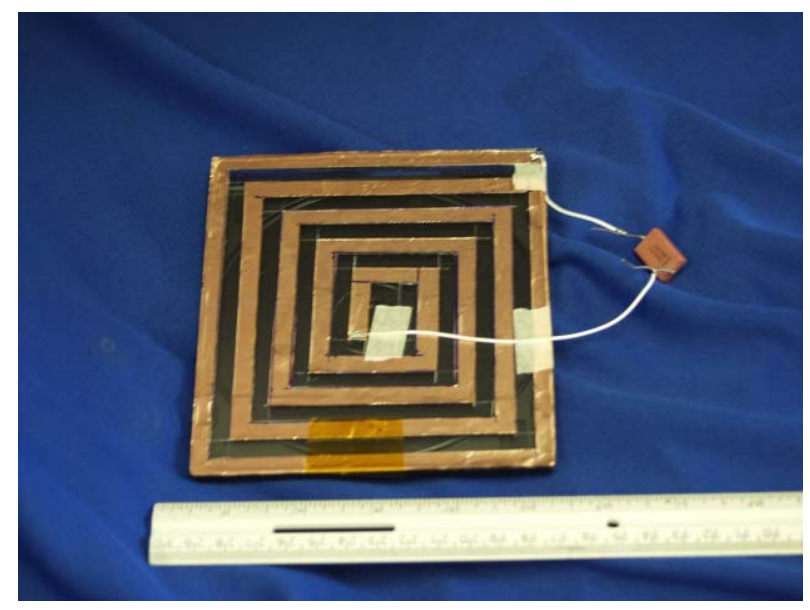

Fig 14 Five-inch square spiral inductor with 0.25 in trace and 0.25 in separation between traces

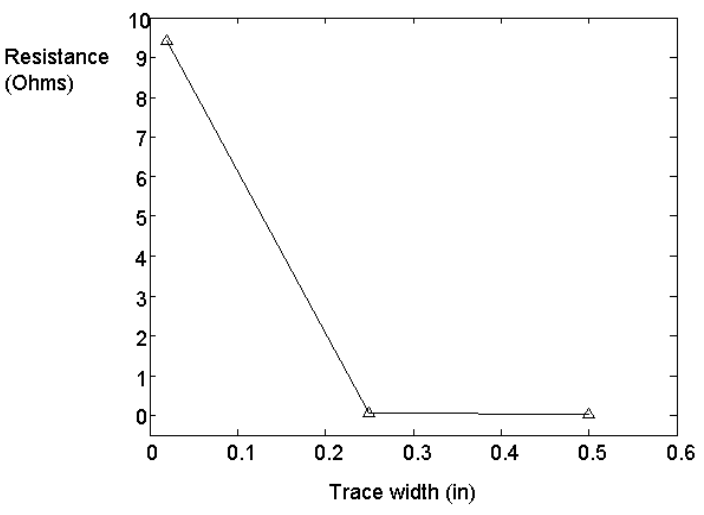

Fig 15 Inductor DC resistance variation with trace width

\section{Concluding Remarks}

A L-C measurement acquisition method has been described. The methodology was developed to increase the distance between an interrogation antenna and a L-C sensor. The method facilitates multiple measurements having different dynamic characteristics. The sweep of a single frequency was used because it concentrates all energy used to excite the L-C sensor into a single harmonic. The L-C sensor response amplitude increases as the excitation harmonic approaches the sensor resonant frequency. The amplitudes are compared to identify the

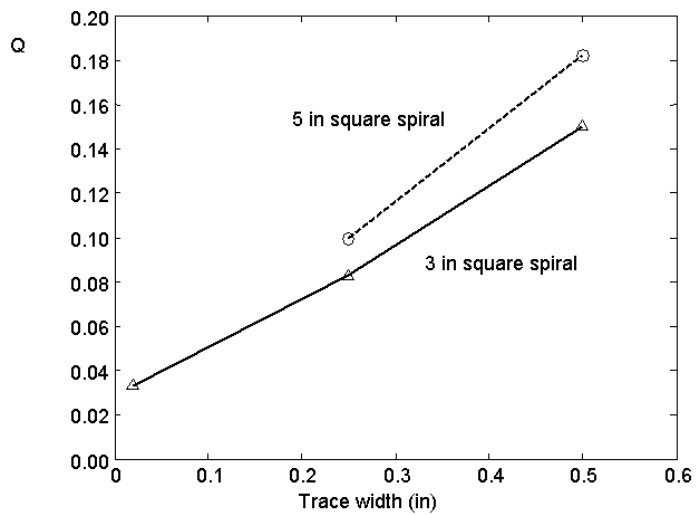

Fig 16 Inductor $Q$ variation with trace width

amplitude inflection. The frequency at which the amplitude inflection occurs is the resonant frequency. The initial sweep is used to ascertain all resonant frequencies and their corresponding amplitudes. Frequencies and amplitude values of successive sweeps can be compared to previous sweeps to ascertain if there is any change to measured property or if the antenna has moved with respect to the antenna. If the physical property has changed, the resonant frequency will be different from prior sweep. If L-C sensor has moved with respect to the antenna, the amplitudes will be different. The magnitude and sign of the difference can be used to determine how fast the sensor is moving and whether 
the sensor is moving toward the antenna or away from the antenna.

Two examples of L-C sensing elements were presented: fluid-level sensor and pressure sensor. The pressure measurement used a fixed harmonic for excitation while pressure was applied. The response amplitude decreased because the excitation frequency remained constant as the resonant frequency changed. Although it was possible to identify the resonant frequency by having the excitation harmonic remain constant while the resonant frequency changed, the lower response amplitude would reduce the distance at which changes in pressure level could be interrogated. The lower response amplitude corroborated the advantage gained from the sweeping harmonic technique. Fluid-level measurements were also presented. A fluid-level of 9 inches resulted in a frequency reduction of over $1 \mathrm{MHz}$ from that of the empty container.

Methods to increase antenna and inductor effectiveness were also presented as techniques to increase the distance between an interrogation antenna and a L-C sensor. A L-C sensor serves as a means of acquiring power via Faraday induction, a sensor and a means of transmitting the measurement via the harmonic magnetic field created by the inductor. The distance at which the magnetic inductor response can be received is proportional to how strong the magnetic field created in the inductor is. The magnetic field strength is dependent upon the current in the sensor. Therefore, interrogation distance is also dependent upon the energy efficiently of the L-C sensor circuit. The higher the energy efficiency, the more current that is created for the same level of power used by the interrogating antenna(e). The quality factor, $\mathrm{Q}$, is the gage for this efficiency. $\mathrm{Q}$ is the ratio of reactance to $\mathrm{DC}$ resistance. A stronger magnetic field is created with higher Q.

Studies were performed to ascertain the effect that antenna width and outer diameter had on DC resistance, inductance and $\mathrm{Q}$. The $\mathrm{DC}$ resistance increases with a longer current path (i.e., due to larger outer diameter) but decreases with increasing width of the path. The geometry of the path also affects the inductance. Measured inductance increased with increasing trace length but decreased as the trace was made wider. The $\mathrm{Q}$ increased with increasing trace width but decreased with increasing trace length. Width has an advantage as a design parameter in that normally, only area dimensions or perimeter dimensions limit size. For any perimeter size, the user can exploit the area within to increase trace width and therefore create more electrically efficient antennae. Q values could possibly be designed using well-known optimization techniques.

\section{References}

1Woodard, S. E., Coffey, N. C., Gonzalez, G. A., Taylor, B. D., Brett, R. R., Woodman, K. L., Weathered, B. W. and Rollins, C. H., "Development and Flight Testing of an Adaptable Vehicle HealthMonitoring Architecture," Journal of Aircraft, Vol 40, No. 5, September-October 2003. Also documented as NASA Technical Memorandum 2003-212139, January 2003.

${ }^{2}$ Halliday, D., and Resnick, R. "Physics Part Two," John Wiley and Sons, New York, NY, 1978, pp.650665 and 770-888.

${ }^{3}$ Lorrain, P. and Corson, D., "Electromagnetic Fields and Waves," W. H. Freeman and Company, 1970, San Francisco, CA, pp. 91-128, and 292-373

${ }^{4}$ Konchin, B., Slavik, I. And Coery, R. W., "Fluid Sensing System," U. S. Patent 6, 335, 690, Jan 1, 2002.

${ }^{5}$ Fonseca, M. A., English, J. M., Arx, M. V. Allen, M. G., "High Temperature Characterization of Ceramic Pressure Sensors," Proceeding of 1999 IEEE MEMS Workshop, pp 146-149.

6Allen, M. A., and English, J. M., "System and Method for the Wireless Sensing of Physical Properties," U. S. Patent 6, 11,520.

Acknowledgments: Dr. R.G. Bryant, R. C. Webster and R. W. Edwards of NASA Langley Research Center; Mr. Rashaan Campbell of Morehouse College and Georgia Institute of Technology. 